\title{
Die Sonnenlampe als Muntermacher des zivilisationskranken Städters
}

\author{
Den Körper regelmässig ultraviolettem Licht auszusetzen, galt nicht immer als \\ gefährlich: Etwa in den 1920er Jahren begann eine Zeit, in der Ärzte genau diese \\ Praktik als Anpassungsmassnahme an die industrialisierte Umgebung empfahlen. \\ Bis zur Jahrhundertmitte entstand ein blühender Markt für Sonnenlampen. Die \\ Behauptung einer Lichtwirkung auf den menschlichen Stoffwechsel war eine Mög- \\ lichkeit, den Nutzen von Ultraviolettbestrahlungen wissenschaftlich zu begründen.
}

\section{Niklaus Ingold}

Mitglied des Graduiertenkollegs am Zentrum «Geschichte des Wissens» (ETHZ/UZH)

1 Quarzlampenvertrieb Zürich. Die Höhensonne (Inserat). Volksgesundheit. 1952; 45(2):41.

2 Michael RA, Ostheimer KG. The evolution of current medical and popular attitudes toward ultraviolet light exposure. Part 2. J Am Acad Dermatol. 2003:48(6):909-18.

3 Lehmann G, Szakáll A. Der Einfluss der Ultraviolettbestrahlung auf den Arbeitsstoffwechsel und die Arbeitsfähigkeit des Menschen. Arbeitsphysiologie. 1932;5: 278-341. Ich zitiere aus diesem Bericht im Folgenden ohne weitere Seitenangaben.

Korrespondenz:

Niklaus Ingold, lic. phil. Forschungsstelle für Sozialund Wirtschaftsgeschichte der Universität Zürich Rämistrasse 64

CH-8001 Zürich

ningold@access.uzh.ch
Um die Mitte des 20. Jahrhunderts bestand in Europa und Nordamerika ein florierender Markt für Ultraviolettstrahler. Firmen wie Solis, Siemens oder Philips stellten sogenannte Sonnenlampen her und bewarben sie für tägliche «Lichtduschen». Anders als heutige Solarien sollten diese Lampen nicht einfach dem Bräu-

\section{Abbildung 1}

Verkäufer bewarben Sonnenlampen in den 1950er Jahren als «Wohltat für die ganze Familie».

\section{Bildnachweis: Heraeus Noblelight $\mathrm{GmbH}$.}

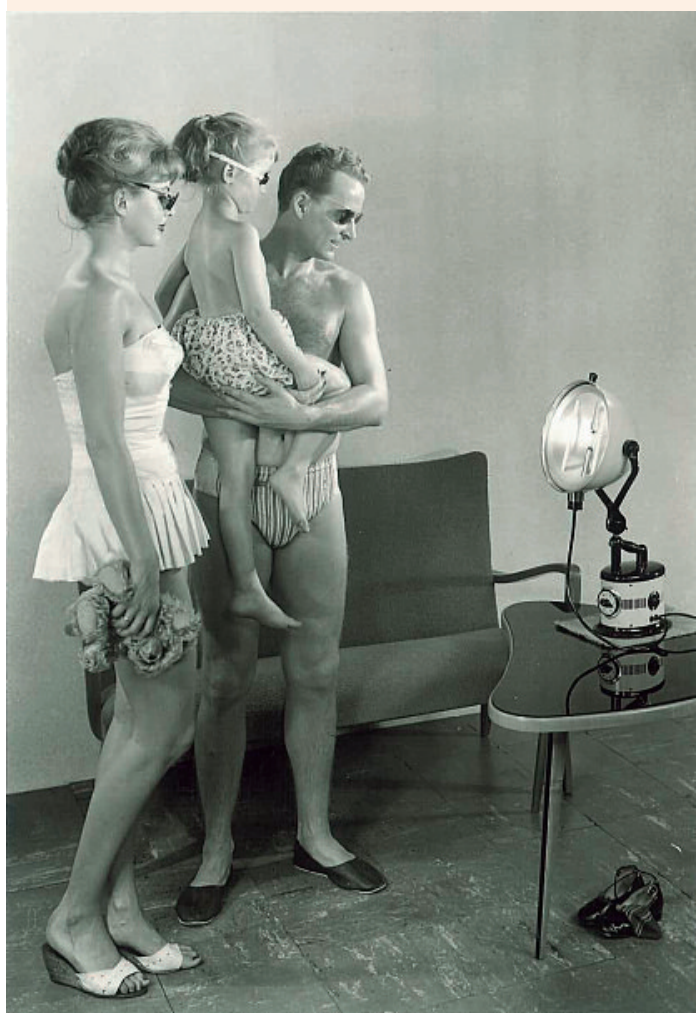

nen des Teints dienen. Sie konnten mehr: «Spendet Lebensfreude und stärkt die Gesundheit - eine Wohltat für die ganze Familie», lautete eine typische Werbebotschaft [1]. Zur Illustration verwendeten die Verkäufer in Inseraten und Prospekten Bilder, die sportliche Frauen, Männer und Kinder oder ganze Familien zu Hause vor Ultraviolettstrahlern zeigen.

Heute wird die Beliebtheit von Sonnenlampen als Mittel zur Gesundheitspflege mit einem spektakulären Behandlungserfolg erklärt: 1919 gelang es Kurt Huldschinsky, Assistenzarzt in einem Berliner Kinderheim, mit einem solchen Apparat Rachitis zu heilen. Diesen Erfolg bei der verbreiteten Kinderkrankheit hätten viele als schlagenden Beweis für die gesundheitsfördernde Wirkung ultravioletter Strahlen betrachtet, lautet das Argument [2]. Jedoch leuchtet nicht ein, weshalb die Genesung kranker Kinder die allägliche Bestrahlung gesunder Erwachsener zur Folge haben sollte.

Ein anderer Erklärungsansatz lässt sich anhand einer Untersuchung veranschaulichen, die zwei Ärzte Anfang der 1930er Jahre am Kaiser-Wilhelm-Institut für Arbeitsphysiologie bei Dortmund durchführten: Gunther Lehmann und Alexander Szakáll wollten klären, ob Bestrahlung mit kurzwelligem Licht die Leistungsfähigkeit steigere [3]. Sie liessen Versuchspersonen auf einem Fahrradergometer in die Pedale treten und massen die geleistete Arbeit. Dazwischen schalteten sie Bestrahlungen mit Sonnenlampen. Drei Merkmale dieser Untersuchung will ich hier hervorheben.

\section{Stadtbevölkerung als medizinische Risikogruppe}

Erstens hatte das kurzwellige Licht eine Bedeutung für die Lebensprozesse im menschlichen Körper. Lehmann und Szakáll gingen von einer Wirkung auf den Stoffwechsel aus. Allerdings merkten sie an, dass «ausser- 
ordentlich wenig Sicheres über den Einfluss der Ultraviolettstrahlen auf den Organismus» bekannt sei. Um mehr über die Lichtwirkung «auf den im klinischen Sinne gesunden Menschen» herauszufinden, massen sie neben der verrichteten Arbeit auch die «Stoffwechselumstimmungen» im Körper.

Zweitens war der Einfluss des Lichts auf den Menschen problematisch: Lehmann und Szakáll verglichen die Lebensbedingungen in den Industriezentren

\section{Abbildung 2}

Bestrahlungen mit ultraviolettem Licht gehörten zu einem Lebensstil, der an die industrialisierte Umgebung angepasst war.

\section{Bildnachweis: Heraeus Noblelight $\mathrm{GmbH}$.}

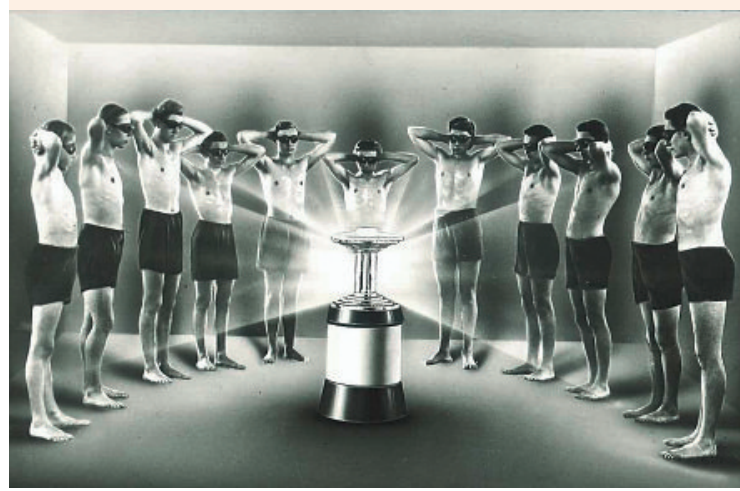
mit denjenigen im Gebirge und nahmen die allerdings nicht genauer bestimmte - Tagesration an kurzwelligem Licht des «Hochgebirgsbewohners» als die gesunde Dosis an. Dieser Vergleich machte die Bewohnerinnen und Bewohner der Industriestädte zu einer medizinischen Risikogruppe: Denn Dampf, Rauch und Staub hüllten laut Lehmann und Szakáll die Industriezentren in eine Dunstglocke, die das kurzwellige Sonnenlicht absorbierte. Die beiden Wissenschaftler diagnostizierten der Stadtbevölkerung deshalb chronischen «Ultraviolettmangel», der anfällig für akute Erkrankungen mache und die Arbeitsleistung vermindere. Mit ihrem Versuch behandelten sie nun ein «hygienisch und volkswirtschaftlich eminent wichtiges Problem».

Schliesslich, drittens, hatten die Arbeitsphysiologen ein ganz bestimmtes Hindernis im Auge, das der Verwendung der Sonnenlampen als Muntermacher für breite Kreise im Weg stand: Die Sportwelt entzweite gerade die Frage, ob Ultraviolettbestrahlungen als Doping zu verbieten seien (vgl. [4]). Diese Debatte war für Lehmann und Szakáll brisant, da der Sportler jene Eigenschaften besass, die der siechen Stadtbevölkerung fehlten: Sein trainierter Körper symbolisierte Lebensfreude, Frische und Beweglichkeit. Die beiden Wissenschaftler wollten deshalb verhindern, dass Sportärzte und -funktionäre Ultraviolettbestrahlungen als Doping einstuften und die Avantgarde der Körperkultur zurückpfiffen.

Ein Doping war der damaligen Definition zufolge ein Mittel, das Höchstleistungen ermöglicht, das aber bei häufiger Anwendung den Körper schädigt. Sollte der Nachweis einer leistungssteigernden Lichtwirkung gelingen, mussten Lehmann und Szakáll folglich negative Nebenwirkungen ausschliessen können, um gegen den Dopingvorwurf zu argumentieren. Zur Beurteilung dieses Kriteriums achteten Lehmann und Szakáll allein auf Veränderungen im Stoffwechsel. Hautkrebs war kein Thema, weil die kanzerogene Wirkung kurzwelliger Strahlen noch nicht als hartes Faktum galt.*

\section{Kein Schaden, Leistungssteigerung um bis zu 60 Prozent}

Ihre Messungen zum Stoffwechsel verglichen Lehmann und Szakáll mit Angaben von Ärzten, die Sportler während des Trainings ohne Bestrahlung untersucht hatten. Die Physiologen stellten fest, «dass die Verschiebungen des Blutbildes durch sportliches Training und durch Ultraviolettbestrahlungen [...] gleich sind». Diese Parallele sprach gegen eine Schädlichkeit der Bestrahlungen. Mit Blick auf die Dopingdebatte folgerten Lehmann und Szakáll: «Ein Mittel, das den Stoffwechsel in einer dem normalen Trainingszustand so ähnlichen Weise beeinflusst, darf wohl nicht als Doping bezeichnet werden.»

Eine theoretische Erklärung für die Parallele hatten die Wissenschaftler keine. Sie vermuteten, dass das Gemeinsame des Trainings und der Bestrahlung in einer «Reizwirkung» liege, «die primär den Eiweissstoffwechsel trifft».

Weniger vage blieben die Angaben zum Leistungsvermögen: Die Arbeitsphysiologen beobachteten eine Erhöhung um bis zu 60 Prozent. Für die alltägliche Verwendung der Sonnenlampen relativierten sie diesen Befund allerdings: «Wir dürfen nicht erwarten, in der Praxis Leistungssteigerungen von $60 \% \mathrm{zu}$ sehen [...], weil man einmal mit der Dosierung vorsichtiger sein muss, und weil zweitens einer so hohen Leistungssteigerung des einzelnen Mannes in der Praxis durch Einflüsse verschiedenster Art entgegengewirkt wird.»

\section{Die Karriere der Sonnenlampen erklären}

Anhand der Zergliederung der Untersuchung am Kaiser-Wilhelm-Institut für Arbeitsphysiologie lässt sich eine bestimmte Vorstellung über die Bedeutung ultravioletter Strahlen für die Lebensprozesse im menschlichen Körper (Stoffwechsel) aufspüren. Solches Wissen inspirierte die Verkäufer der Sonnenlampen zu Werbebotschaften wie «Spendet Lebensfreude und stärkt die Gesundheit ...». Gleichzeitig wird deutlich, dass die Empfehlung täglicher «Lichtduschen» mit einem komplexen Gefüge von Einflussfaktoren verbunden war: Im beschriebenen Beispiel spielte neben der bestimmten Vorstellung über die Lichtwirkung auf den Menschen eine bestimmte Art, diese Lichtwirkung zu problematisieren (Ultraviolettmangel), eine Rolle, und es galt ein Hindernis (Dopingvorwurf) zu überwinden.

Derartige Gefüge waren die Bedingungen für das Auftauchen von Apparaten zur Bestrahlung des Körpers an unterschiedlichen Orten in der Gesellschaft. Die Gefüge entstanden, veränderten sich und fielen auseinander. Anstatt spektakuläre Behandlungserfolge $\mathrm{zu}$ einer Geschichte der Lichttherapie aufzureihen, können diese Prozesse beschrieben werden. Letztlich wird dann die Entstehung und Veränderung jenes Wissens beobachtet, das das nach wie vor gültige Ideal des gebräunten, trainierten Körpers mitgeformt hat.

\footnotetext{
Wiederholung eines solchen Nachweises bereitete den Wissenschaftlern jedoch Mühe. Vgl. [5].

George Marshall Findlay wies einen kausalen Zusammenhang zwischen ultravioletten Strahlen und Hautkrebs bei Albinomäusen nach. Die

4 Hoberman JM. Mortal
Engines: The Science of Performance and the

Marshall Findlay G. Ultraviolet light and skin cancer. Lancet. 1928;212(2):1070-3.
} 